\title{
Molecular diagnosis and inborn errors of metabolism: A practitioner's view
}

\author{
Stephen Cederbaum, MD
}

\begin{abstract}
Recombinant DNA technology has altered completely the face of genetics and its clinical practice. cDNAs for enzymes involved in inborn errors were among the first cloned, but the impact of this advance on our subspecialty has been more limited. This discussion addresses the uses and "abuses" of recombinant DNA technology in the field, and areas of greater or lesser usefulness in current practice, and the future, are discussed. Genetics in
\end{abstract} Medicine, 2000:2(6):345-349.

Editor's Note: This short paper was delivered at the joint meeting of the Society for Inherited Metabolic Disorders and the American College of Medical Genetics. It is the third paper from this joint symposium and represents the thoughtful opinion of a senior established practitioner in metabolic diseases, and it is meant to be provocative. The Editor-in-Chief encourages College members and others to respond and indicate their views on this important issue.

It was nearly 20 years ago that the Society for Inherited Metabolic Disorders held a symposium to discuss the emerging field of recombinant DNA in medicine. Insulin, growth hormones, somatostatin, $\alpha_{1}$-antitrypsin, and a number of other circulating molecules were the focus of attention. Phenylalanine hydroxylase and ornithine transcarbamylase were the lone $\mathrm{CDNAs}$ for the enzymes involved in inborn errors that had been cloned, but work on them had not progressed to the point of achieving a place on the program., ${ }^{1,2}$

It would be an understatement to say that gene cloning has revolutionized the practice of medical genetics. The genetic bases of innumerable disorders have been elucidated with the positional cloning of their genes. Confirmation of a clinical diagnosis is possible in many instances by finding significant mutations in the appropriate gene. The issue has become more one of cost and availability than possibility.

Ironically, despite the fact that the genes for the enzymes involved in different inborn errors had been among the first cloned, the effect on the field has been less revolutionary. The reason is quite apparent: for many, if not most of the inborn errors of metabolism, the enzyme involved is known and its deficiency can generally be documented by an enzymatic assay. Understanding the disorder and its pathogenesis is more de-

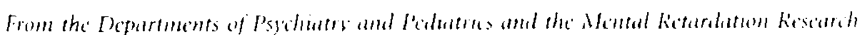
Conter LCLA, I Is dengeles, Cilliformat

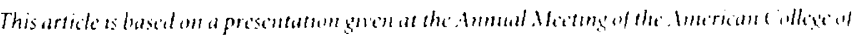
Medical Gentrics, March 2000). Palm Sprmgs. Cuhtornia.

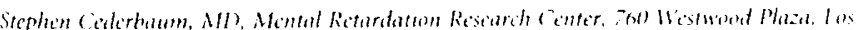

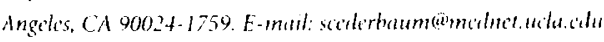

Recietied: September 25, 2000.

Accipted: Nowember 1, 2000 .
}

pendent on the nature and level of the accumulated or deficient metabolites than it is on the specific gene mutation. The next great frontier in inborn errors, understanding and interrupting the pathogenic process, may be completely dependent on knowledge of the metabolites, although their effect on other genes and their products may ultimately be extremely important as well.

Having made these introductory observations, the remainder of this essay will explore the role that DNA analysis is playing in the field of inborn errors of metabolism, areas in which it may prove useful and others in which its usefulness has proven to be disappointing and essentially useless. In some instances the results were predictable.

Some opening qualifications are in order. These observations are meant to be provocative, if for no other reason than genotyping patients and the dreaded phrase, genotype-phenotype correlation, have been overly touted for a decade. These comments are meant to try to define the clinical usefulness of DNA analysis and will not address its importance in research. Finally, these observations are based on the state of the art today or in the foreseeable future. Technology or insights not now apparent may alter the landscape considerably.

\section{Consensus areas}

There are a number of areas in which both enthusiasts and skeptics would agree. These are listed in Table 1. These uncontroversial areas will be discussed only briefly.

The first is in prenatal diagnosis, especially if the substrate levels in the amniotic fluid or enzyme assays in either chorionic villus biopsies or amniotic fluid cells are unreliable predictors of affected or normal fetuses. This can be carried out either by linkage to easily assessed markers, or now more frequently by gene or CDNA sequence analysis. In either instance these are labor-intensive and uncertain, and planning must occur in advance of the pregnancy.

A second area of consensus is the determination of carrier status of an individual. This is equally important in X-linked disorders such as Fabry disease or ornithine transcarbamylase deficiency, both instances in which reliable clinical or clinical 
Table 1

Areas of consensus between skeptics and enthusiasts of the roles of DNA analysis in inborn errors of metabolism

\begin{tabular}{l}
\hline Consensus \\
\hline Prenatal diagnosis \\
Linkage \\
Sequence-expensive \\
- Carrier status \\
- Research \\
$\quad$ Enzyme structure \\
Cellular effects of error \\
- Demographic history \\
- Gene therapy \\
\hline
\end{tabular}

biochemical indicators are scarce. For autosomal disorders, these methods are in most prominent use for carrier testing in populations in which a disease incidence is high and the number of mutant alleles low. Examples would be screening for Ashkenazi Jewish disorders, tyrosinemia in French Canadians and hemoglobinopathies in certain at-risk populations, and others.

A third area is research into enzyme structure and function. A missense mutation can be placed in the crystal structure of an enzyme and the impact predicted with a considerable degree of probability (for example, Ash et al. ${ }^{3}$ ). In some few instances, mutation analysis may predict the cellular effects of the aberrant RNA or gene product. An example might be $\alpha_{1}$-antitrypsin deficiency in which the common $\mathrm{Z}$ mutation is most associated with liver disease. ${ }^{4}$

Mutation and haplotype analysis may be used successfully to trace the origin of a mutation and its population movements. This would rarely be useful in the clinical context. It might be lumped with curiosity as poor clinical reasons for undertaking DNA analysis of a gene whose product is deficient in an inborn error.

Finally, I want to mention gene therapy. Recent publicity suggests that this may be on "life support." Most of us believe, however, that in the future it will become a reality - a critical intervention in many inborn errors and a cure in others. Depending on the eventual technology, knowledge of the specific mutation may be important. This paper, however, is not the context in which to address gene therapy.

\section{Areas of no consensus}

In reality, many aspects of the role of DNA in inborn errors lack, at this time, a consensus (Table 2). Sometimes the disagreement hinges on economic and technical issues such as who will pay for the study. If funds are limited, is the cost benefit sufficient? If it is justified on a cost-benefit basis, how can we "get it done," even if funds are available? We often
Table 2

Areas lacking consensus about the importance of DNA analysis in inborn errors of metabolism

No Consensus
Drimary diagnosis
Role in unknown disorders
Prognosis and treatment
Phenotype-genotype correlation
Population screening
- Role when there is predominant mutation

imagine that if we can get the test paid for, all is well. We forget that it is far more difficult to receive reimbursement for the people who must obtain authorization for payment for these arcane, out-of-state tests, unknown to the mainstream clinical pathology or the third-party payer communities.

In this discussion I will choose as examples, particular higher-profile disorders, recognizing that others may present equally compelling models.

Genotype analysis, or searching for mutations, has an important role in disorders previously defined by phenotype alone. In these disorders a DNA abnormality may be the only "proof" for the accuracy of the diagnosis and the establishment of the genetic and clinical prognosis. What, however, is the role and use of DNA mutation (or other) analysis in the diagnosis of inborn errors?

The first example would be one in which either newborn screening or metabolite analysis in a patient at higher risk revealed a minor but persistent deviation from the established norms. An example might be a minor elevation of methylmalonate or propionate on a newborn screening study by tandem mass spectrometry. ${ }^{5,6}$ Currently we might depend on conventional biochemistry and repeated studies when the child is ill. Less frequently some might use a provocative test or a "challenge." In principle, one could propose sequencing one, several, or all of the genes in the pathway. In the future this might prove more accessible with the advent of the gene chip. For the present and the foreseeable future it remains too inaccessible and too expensive. Whether the gene chip proves to be a panacea is problematic because its absolute reliability is unproven and the mutation could lie outside the studied region, as is the case in some instances now. Finally, "mild mutations" could lie in amino acids whose importance cannot be easily inferred, leading to an ambiguous result. DNA is probably not the answer here.

The most frequent instance in which primary DNA analysis is recommended for diagnosis is in cases of suspected mitochondrial disorders. The rationale and the problems with this approach are outlined in Table 3. Although the majority of proteins in the mitochondrion are encoded in the nuclear genome, mutations in the 13 structural proteins and the unique mitochondrial tRNA genes and deletions and/or duplications 
Table 3

Rationale and problems in the use of DNA analysis in the diagnosis of suspected disorders of the mitochondrial respiratory chain

Energy metabolism

- Mitochondrial genome

- Tissue disparity

- Low prior probability

- Polymorphisms

- Cost

Missed cases

of the mitochondrial genome of 16,564 base pairs are found in disproportionate frequency in proven disorders of energy metabolism. ${ }^{7}$ In cases in which an error of energy metabolism is probable, study of DNA of the mitochondrial genome either in blood, and especially in an affected organ such as muscle, is a rational and frequently rewarding exercise. In these situations the important clinical implications justify the expense of the testing.

This pleasant picture is clouded, however, by the current state of medical practice with regard to these disorders and ironically, by advancing technology. Because the manifestations of mitochondrial disorders are protean and infrequently are responsible for isolated single-organ involvement in diseases such as diabetes or deafness, many disorders without proven etiology and unlikely to be due to mitochondrial abnormalities have mitochondrial disease added to the differential diagnostic list. ${ }^{7}$ Mitochondrial DNA mutation analysis, quite cost-effective when used appropriately, becomes an expensive and quite useless indulgence.

Unfortunately the advancing technology is likely to make the situation worse. Already, manual methods for screening the entire mitochondrial genome for mutations are available, albeit expensive and slow. In instances with lower prior probability of a mitochondrial mutation, polymorphisms creating false-positive results will far exceed in frequency meaningful mutations. Efforts to sort through this morass involve costly and risky interventions. The advent of the mitochondrial gene chip will only add to this the technical uncertainty of amplification and hybridization. The polymorphisms, the technical errors, and the computer programs to analyze and interpret the results will create an expensive cottage industry.

Whether the gene chip, which eventually will be applied to other disorders, will be a blessing or a curse remains to be determined. My vote at the moment is cast for curse. I am concerned with polymorphisms, and especially misleading results from the technology.

DNA analysis in inborn errors as a prognostic indicator has been a major disappointment in most instances, although no major surprise to many. To be truly useful and acceptable, the method would have to replace metabolite measurement or alter in some way treatment and prognosis independently of metabolite or dietary information. Consideration of the extensive mutation data in the hyperphenylalaninemias is illustrative (Table 4).

More than 350 mutations "causing" the hyperphenylalaninemias have been determined. Many of the missense mutations have been expressed in Escherichia coli. In the main there is a reasonably good correlation between the "severity" of the mutation and the degree of phenylalanine intolerance, as one would expect. Nevertheless, the correlation is imperfect and none of the data allow one to forego the current empirical methods of measuring the effect of dietary restriction of different degrees with the plasma phenylalanine level.8.9 The impact of other genes and the environment are known to vary plasma phenylalanine levels in siblings and others with identical mutations. Moreover, the same level of phenylalanine in plasma may have widely different effects on the brain in different people. Genotyping has not proven to be clinically useful in this family of disorders.

The same can be said for another disorder screened in most newborn programs, galactosemia. Data indicate that individuals with the most common Q188R mutation may have a worse outcome than patients with many other mutations. ${ }^{10}$ Few, however, would be brave enough, despite the statistical correlations, to doom these homozygotes or convey optimism to those with many other mutations. In fact, this information might tempt us to open our mouths when we should not.

Despite these examples of a general phenomenon, there are exceptions to the rule. One example would be Gaucher disease of early onset in a non-Ashkenazi Jewish person with no neurological findings. The presence of a "protective" allele such as N370S would greatly diminish the likelihood of the neurologic manifestations that might be anticipated otherwise. "

Two other exceptions would be population screening for the methylene tetrahydrofolate reductase $677 \mathrm{C}-\mathrm{T}$ polymorphism and carrier testing for many X-linked disorders. In the former case, DNA analysis is probably the most effective means for population screening for carrier status. ${ }^{12}$ In other recessive disorders, if the population group screened has a preponderance of one or several mutations, carrier testing can be most effectively carried out by these means. This approach is used for "the Jewish genetic diseases" and is recommended for cystic fibrosis follow-up in newborn screening programs. ${ }^{12-14}$ The

Table 4

Experience with DNA analysis in the hyperphenylalaninemias PKU experience

$>350$ mutations defined

- Many missense expressed in E. coli

- Reasonable correlation with "severity"

Imperfect predictor

Of little clinical use

PKU, phenylketonuria. 
consensus seems to be that this is premature in other instances, although the debate is fierce for hemochromatosis, which may be considered an inborn error. If screening for hemochromatosis were to be undertaken, would DNA screening be more efficient than the periodic screening of ferritin?

When diseases have a predominant mutation as their cause, the use of mutation analysis may be a powerful adjunct or substitute for biochemical analysis, particularly for disorders in which the clinical biochemical perturbations are intermittent, the enzyme assay in accessible tissues is difficult and treacherous, or the enzyme is expressed only in a visceral and hence less accessible tissue. An example of this might be medium-chain acyl CoA dehydrogenase deficiency or more particularly long-chain hydroxy acyl CoA dehydrogenase deficiency. ${ }^{15}$ It is important to bear in mind, however, that a negative result in such an assessment is far less meaningful than a positive one.

An example of the use of DNA analysis in this way occurred in one of our own patients. An infant had all of the clinical features of glycogen storage disease, type I, but the glucose-6phosphatase activity in a liver biopsy specimen was normal. The finding of a known mutation in one of his two glucose-6phosphatase alleles allowed us to infer, with a high degree of confidence, that our clinical impression was correct.

\section{Final comments}

When used properly, DNA analysis is a powerful, useful, and discriminating tool for the diagnosis and care of individuals who have or may have an inborn error of metabolism (Table 5). It has been a revolutionary element in our field and will continue to be so in the future. DNA analysis is, however, no panacea. I am concerned that it may have been oversold by many people who feel that their area of interest will be illserved if they are not participants in the DNA revolution. This may have happened in the phenylketonuria field. Furthermore, the indiscriminate use of DNA analysis may be a costly adjunct to a field already underfunded, and in the worst-case scenario, it may give the illusion of understanding that does not exist.

\section{Table 5}

State of the field

Final comments

- DNA analysis is a powerful and useful tool

DNA is no panacea

- DNA analysis will not rescuc imprecise thinking

- With exceptions, DNA analysis will be expensive

- Too much DNA--too many polymorphisms

- DNA analysis will miss large deletions

DNA is not the last frontier
DNA analysis will not rescue imprecise thinking, nor will it usually bail one out of a diagnostic dilemma. New disorders may be discovered in this way, but this approach is generally too cost-inefficient to justify routine clinical use, at least under the constraint currently extant in U.S. medicine. The use of DNA analysis has been particularly prevalent in possible disorders of energy metabolism, but here too, as the technologies expand, the efficiency will continue to fall. It is simply impossible and unreasonable to use DNA technology indiscriminately. Too great a lowering of prior probability will raise the prevalence of false-positive results unacceptably.

The expense of widespread testing will be great. The gene chip, when in more prevalent use, may reduce unit costs, but it will never be cheap. Any cost savings will be eliminated quickly by the expanded net its availability may tempt people to cast.

Nor will the gene chip be precise. The number of steps involved, and the inherent variability at each step, will yield falsepositive, false-negative, and truly ambiguous results. If you examine thousands of hybridizations, you don't need many false results to undermine completely the intellectual and economic foundations of genetic practice. Moreover, human polymorphisms will yield false results even when the analytical results are accurate. Large deletions may be missed as well without elaborate internal controls that in the end may not be fully realizable. We cannot allow the gene chip to be the automated karyotype, a technology that has largely been a failure despite great efforts and much optimism.

Finally, I would like to emphasize my belief that DNA is far from the last frontier in inborn errors. The normal and abnormal physiology for many of the biochemical pathways are poorly known and may be far more important to the diagnosis and care of our patients than is their mutation. Modalities such as positron emission tomography and magnetic resonance spectroscopy may be the tip of the useful diagnostic iceberg.

\section{Acknowledgments}

Supported by the Mental Retardation Research Program at UCLA and by USPHS Grants HD-06576, HD-36415, and HD04612. The author thanks Eric Vilain and Katrina Dipple for reading and critiquing early drafts of this presentation. They sharpened the focus and eliminated outrageous hyperbole.

\section{References}

1. Robson KJH, Chandra T, MacGillivray RTA, Woo SLC. Polysome immuno-precipitation of phenylalanine hydroxylase mRNA from rat liver and cloning of its cDNA Proc Natl Acad Sci U S A 1982;79:4701-4705.

2. Horwich AL, Kraus JP, Williams KR, Kalousek F, Konigsberg W, Rosenberg LE. Molecular cloning of the CDNA coding for rat ornithine transcarbamylase. Proc Nat Acad Sci U S A 1983;80:4258-4262.

3. Ash DE, Scolnick LR, Kanyo ZF, Vockley IG, Cederbaum SD, Christianson DW Molecular basis of hyperargininemia: structure-function consequences of mutations in human liver arginase. Mol Gent Metab 1998;64:243-249.

4. Cox DW. $\alpha$-Antitrypsin deficiency. In: Scriver CR, Beaudet AL, Sly WS, Valle D, editors. The metabolic and molecular bases of inherited disease. New York McGraw-Hill, 1995:4125-4158.

5. Sniderman LC, Lambert M, Giguere R, Auray-Blais C, Lemieux B, LaFramboise R, 
Rosenblatt DS, Treacy EP. Outcome of individuals with low-moderate methylmalonic aciduria detected through a neonatal screening programs. I Pediatr 1999;134: 675-680.

6. Cederbaum S, Vilain E. Newborn screening is going to expand: are we ready? [Editorial] J Pediatr 1999;133:666-667.

7. Shoffner IM, Wallace DC. Oxidative phosphorylation diseases. In: Scriver CR, Beau$\operatorname{det} \mathrm{AL}$, Sly WS, Valle D, editors. The metabolic and molecular bases of inherited disease, 7th ed. New York: McGraw-Hill, 1995:1535-1609

8. Enns GM, Martinez DR, Kuzmin AI, Koch R, Wakeem CK, Woo SL, Eisensmith RC, Packman S. Molecular correlations in phenylketonuria: mutation patterns and corresponding biochemical and clinical phenotypes in a heterogeneous California population. Pediatr Res 1999;46:594-602.

9. Scriver CR, Waters PJ. Monogenic disorders are not simple: lessons from phenylketonuria. Trends Genet 1999;15:267-272.

10. Robertson A, Singh RH, Guerrero NV, Hundley M, Elsas LJ. Outcomes analysis of verbal dyspraxia in classic galactosemia. Genet Med 2000;2:142-148.
11. Beutler E, Grabowski GA. Gaucher disease. In: Scriver CR, Beaudet AL, Sly WS, Valle $D$, editors. The metabolic and molecular bases of inherited disease, 7 th ed. New York: McGraw-Hill, 1995:2641-2670.

12. Eng CM, Schechter C, Robinowitz J, Fulop G, Burgert T, Levy B, Zinberg R, Desnick RJ. Prenatal genetic carrier testing using triple disease screening. JAMA 1997;278: $1268-1272$

13. Hartge P, Struewing IP, Wacholder S, Brady LC, Tucker MA. The prevalence of common, BRCAl and BRCA2 mutations among Ashkenazi Jews. Am I Hum Genet 1999;64:963-970.

14. Gregg RG, Simantel A, Farrell PM, Koscik R, Kosorok MR, Laxova A, Laessig R, Hoffman G, Hassemer D, Mischler EH, Splaingard M. Newborn screening for cystic fibrosis in Wisconsin: comparison of biochemical and molecular methods. Pediatrics 1997:99:819-824

15. Ibdah JA, Bennett MI, Rinaldo P, Zhao Y, Gibson B, Sims H, Strauss AW. A fetal fatty acid oxidation disorder causes maternal liver disease of pregnancy. $N$ Engl J Med 1999;340:1723-1731 